\title{
Long-Distance Asymptotics of Temperature Correlators of the Impenetrable Bose Gas
}

\author{
A. R. Its ${ }^{1}$, A. G. Izergin ${ }^{2 *}$ and V. E. Korepin ${ }^{3}$ \\ ${ }^{1}$ Leningrad University, Leningrad, USSR \\ 2 International Centre for Theoretical Physics, Trieste, Italy \\ ${ }^{3}$ Leningrad Branch, V. A. Steklov Mathematical Institute, Fontanka 27, SU-191011 Leningrad, USSR
}

\begin{abstract}
The inverse scattering method is applied to the integrable nonlinear system describing temperature correlators of the impenetrable bosons in one space dimension. The corresponding matrix Riemann problems are constructed for two-point as well as for multi-point correlators. Long-distance asymptotics of two-point correlators is calculated.
\end{abstract}

\section{Introduction}

Impenetrable Bose gas in one space dimension representing bosons with the pointlike infinite repulsion is the simplest nontrivial integrable model. In the state of the thermal equilibrium at temperature $T>0$ the distribution of particles with momenta $k$ is given by the Fermi weight $(1+\exp \{\varepsilon(k) / T\})^{-1}$ (energy $\varepsilon(k)=k^{2}-h, h$ is a chemical potential). Our aim is to obtain the long-distance asymptotics of equal-time temperature correlators in the model. To do this we use essentially results of a previous paper [1], where the completely integrable system describing temperature correlators was constructed and partial differential equations for them were obtained. So the description of temperature correlators in the quantum model is reduced to the investigation of the classical nonlinear integrable system. This is done by means of the inverse scattering method in the matrix Riemann problem formulation (see, e.g. [2]).

Let us begin with introducing notations and formulating results of paper [1] further. The two-point correlators will be mainly considered, namely, the two-point field correlator

$$
\left\langle\psi^{+}(z) \psi(-z)\right\rangle_{T}=\left.(\sqrt{T} / 4) B_{++}(x, t, \gamma) \Delta(x, t, \gamma)\right|_{\gamma=2 / \pi}
$$

\footnotetext{
$\star$ Permanent address: Leningrad Branch, V. A. Steklov Mathematical Institute, Fontanka 27, SU-191011 Leningrad USSR
} 
and the generating functional for density correlators

$$
\left\langle\exp \left\{\alpha \int_{0}^{z} \psi^{+}(y) \psi(y) d y\right\}\right\rangle_{T}=\left.\Delta(x / 2, t, \gamma)\right|_{\gamma=(1-\exp \{\alpha\}) / \pi}
$$

The value of the last correlator at $\alpha=-\infty(\gamma=1 / \pi)$,

$$
P(z, h, T)=\left.\Delta(x / 2, t, \gamma)\right|_{\gamma=1 / \pi}
$$

gives the probability that there are no particles with coordinates $0 \leqq y \leqq z$ at the state of the thermal equilibrium ("emptiness formation probability") and is of special interest.

On the right-hand sides of representations (1.1)-(1.3) $x$ and $t$ are the renormalized distance and the chemical potential,

$$
x=z \sqrt{T}, \quad t=h / T,
$$

and $\Delta$ is the Fredholm determinant,

$$
\Delta(x, t, \gamma)=\operatorname{det}(1-\gamma \mathscr{K})
$$

of the linear integral operator $\mathscr{K}$,

$$
\begin{aligned}
(\mathscr{K} f)(\lambda) & =\int_{-\infty}^{\infty} K(\lambda, \mu) f(\mu) d \mu \\
K(\lambda, \mu) & =[2 i(\lambda-\mu)]^{-1}\left(e_{+}(\lambda) e_{-}(\mu)-e_{-}(\lambda) e_{+}(\mu)\right) .
\end{aligned}
$$

Functions $e_{ \pm}$are

$$
e_{ \pm}(\lambda)=\sqrt{\vartheta(\lambda)} \exp \{ \pm i \lambda x\}
$$

the Fermi weight $\vartheta(\lambda)$ being given as

$$
\vartheta(\lambda)=\left(1+\exp \left\{\lambda^{2}-t\right\}\right)^{-1}
$$

(the dependence of these quantities of $x$ and $t$ will as a rule be suppressed in notations).

"Potential" $B_{++}$in (1.1) is one of the potentials $B_{l m}(l=+,-; m=+,-)$,

$$
\begin{gathered}
B_{l m}(x, t, \gamma)=\gamma \int_{-\infty}^{\infty} e_{l}(\lambda) f_{m}(\lambda) d \lambda, \\
B_{++}^{*}=B_{++}=B_{--} ; B_{+-}^{*}=B_{+-}=B_{-+} .
\end{gathered}
$$

Functions $f_{ \pm}$are the solutions of the linear integral equations

$$
f_{ \pm}(\lambda)-\gamma \int_{-\infty}^{\infty} K(\lambda, \mu) f_{ \pm}(\mu) d \mu=e_{ \pm}(\lambda)
$$

The logarithmic derivatives of the Fredholm determinant $\Delta$ can also be expressed in terms of the potentials $B$. Introducing the function $\sigma$,

one has

$$
\sigma(x, t, \gamma)=\ln \Delta(x, t, \gamma) ; \sigma(0, t, \gamma)=0, \quad \sigma(x,-\infty, \gamma)=0,
$$

$$
\partial_{x} \sigma=-B_{+-}, \quad \partial_{t} \sigma=-x \partial_{t} B_{+-}+(1 / 2)\left(\partial_{t} B_{+-}\right)^{2}-(1 / 2)\left(\partial_{t} B_{++}\right)^{2} .
$$


So all the correlators are represented in terms of the solutions of system (1.10).

It was shown in [1] that the following relation for the two-component function $F(\lambda)\left(F_{1}=f_{+}(\lambda), F_{2}=f_{-}(\lambda)\right)$ are valid,

$$
\begin{aligned}
\partial_{x} F(\lambda) & =\left(i \lambda \sigma_{3}+B_{++} \sigma_{1}\right) F(\lambda), \\
\left(2 \lambda \partial_{t}+\partial_{\lambda}\right) F(\lambda) & =\left(i x \sigma_{3}-i \partial_{t} B_{+-} \sigma_{3}-\partial_{t} B_{++} \sigma_{2}\right) F(\lambda) .
\end{aligned}
$$

This is a zero curvature representation for a nonlinear evolutionary system for potentials $B$,

$$
\partial_{x} B_{+-}=B_{++}^{2}, \quad 2 x B_{++}+\partial_{x} \partial_{t} B_{++}=2 B_{++} \partial_{t} B_{+-},
$$

which results in the equation for the potential $B_{++}$,

$$
\partial_{t}\left(B_{++}^{2}\right)=1+(1 / 2) \partial_{x}\left(\left(\partial_{x} \partial_{t} B_{++}\right) / B_{++}\right)
$$

It should be mentioned that all the results (1.5)-(1.18) are valid not only for function $\vartheta(\lambda)$ defined in (1.8) but also for arbitrary $\vartheta(\lambda)$ satisfying conditions

$$
\left(2 \lambda \partial_{t}+\partial_{\lambda}\right) \Re(\lambda)=0 ;\left.\vartheta\right|_{\lambda^{2}-t \rightarrow+\infty} \rightarrow 0,
$$

this arbitrary function playing a role of a reflection coefficient in the inverse scattering method. So all the notations and necessary results of [1] are given.

The contents of this paper are as follows. We begin with constructing in Sect. 2 the basic ingredient of the inverse scattering method, i.e. the matrix Riemann problem. Linear equations (1.10) (which were the main object of the analysis in [1]) appear to have the sense of simple algebraic reductions for initial data of the special type (1.8), (1.16) of the Gel'fand-Levitan-Marchenko equations. This allows us to calculate in the next sections the long-distance asymptotics of correlators. To do this, we use the scheme of the asymptotic analysis of solutions of nonlinear integrable equations suggested in paper [3]. This is done for correlator (1.2) with $\gamma<1 / \pi$ in Sect. 3. In Sect. 4, the complete asymptotic expansion for the emptiness formation probability $(\gamma=1 / \pi)$ is constructed.

In Sect. 5, the long-distance asymptotics of the two-point field correlator (1.1) is obtained. In the Appendix, the matrix Riemann problem for multi-point correlators is constructed.

\section{Matrix Riemann Problem for Two-Point Correlators}

Consider on the complex plane $\lambda$ the following matrix Riemann problem (MRP). One has to find that the $2 \times 2$ matrix-valued function $\chi(\lambda)(\operatorname{det} \chi(\lambda) \neq 0$ at any $\lambda)$, which is holomorphic for $\operatorname{Im} \lambda>0$ and $\operatorname{Im} \lambda<0$, is equal to the unit matrix $I$ at $\lambda=\infty$,

$$
\chi(\infty)=I
$$

and the boundary values $\chi^{+}(\lambda)$ and $\chi^{-}(\lambda)$ on the real axis are related as

$$
\begin{aligned}
& \chi^{-}(\lambda)=\chi^{+}(\lambda) G(\lambda)(\operatorname{Im} \lambda=0), \\
& \chi^{ \pm}(\lambda)=\lim \chi(\lambda \pm i \varepsilon)(\varepsilon>0, \varepsilon \rightarrow 0) .
\end{aligned}
$$


The conjugating matrix $G$ here is

$$
G(\lambda)=\left(\begin{array}{cc}
1+\pi \gamma e_{+}(\lambda) e_{-}(\lambda) ; & -\pi \gamma e_{+}^{2}(\lambda) \\
\pi \gamma e_{-}^{2}(\lambda) ; & 1-\pi \gamma e_{+}(\lambda) e_{-}(\lambda)
\end{array}\right),
$$

functions $e_{ \pm}(\lambda)=\sqrt{\vartheta(\lambda)} \exp \{ \pm i \lambda x\}$ and $\vartheta(\lambda)=\left(1+\exp \left\{\lambda^{2}-t\right\}\right)^{-1}$ are the same as in (1.7) and (1.8).

The explicit form of the matrix $G$ allows us to use the classical results (see e.g. [4]) to show that this MRP is uniquely solvable at any $x$ and $t$ if

$$
-1 / \pi \leqq \gamma \leqq / \pi
$$

and that the solution $\chi(\lambda)$ is a smooth function of parameters $x, t$. If condition (2.4) is violated, the question of solvability becomes nontrivial. However, the sufficient conditions of solvability are also fulfilled, e.g. in the case

$$
\gamma=2 / \pi, \quad-\infty<x<+\infty, \quad t<0 .
$$

Supposing that the MRP is solvable, let us study the properties of the solution as a function of $x, t$. It should be noted that at $|\lambda| \rightarrow \infty$, function $\chi(\lambda)$ can be expanded as

$$
\chi(\lambda)=I+\left(\Psi_{1} / \lambda\right)+\left(\Psi_{2} / \lambda^{2}\right)+\cdots,
$$

where functions $\Psi_{i}(x, t)$ do not depend on $\lambda$. Due to the symmetry properties of matrix $G$,

$$
G(\lambda)=\sigma_{1} G^{-1}(-\lambda) \sigma_{1} ; \quad G(\lambda)=G^{*}(-\lambda),
$$

one concludes that the coefficient $\Psi_{1}$ can be written as

$$
\Psi_{1}=(-1 / 2) B_{++} \sigma_{2}-(i / 2) B_{+-} \sigma_{3},
$$

where $B_{++}, B_{+-}$are some real scalar functions of $x, t$ (it will be shown later that they are just the potentials (1.9)).

Our first aim is to show that function

$$
\Psi(\lambda)=\chi(\lambda) \exp \left\{i \lambda x \sigma_{3}\right\}
$$

is a matrix solution of a linear system similar to the zero curvature representation (1.13). To this end one rewrites (2.2) as $\Psi^{-}(\lambda)=\Psi^{+}(\lambda) G_{0}(\lambda)$, where matrix $G_{0}$ is

$$
G_{0}(\lambda)=\left(\begin{array}{cc}
1+\pi \gamma \vartheta(\lambda) ; & -\pi \gamma \vartheta(\lambda) \\
\pi \gamma \vartheta(\lambda) ; & 1-\pi \gamma \vartheta(\lambda)
\end{array}\right) .
$$

This matrix does not depend on $x$ and has the property $\left(2 \lambda \partial_{t}+\partial_{\lambda}\right) G_{0}=0$ due to (1.16). Thus the corresponding logarithmic derivatives have no cuts at the real axis and are holomorphic for any $\lambda$. From (2.5) and (2.6) one has then (using the Liouville theorem) that for any $\lambda$

$$
\begin{aligned}
\left(\partial_{x} \Psi\right) \Psi^{-1} & =i \lambda \sigma_{3}+B_{++} \sigma_{1} \\
{\left[\left(2 \lambda \partial_{t}+\partial_{\lambda}\right) \Psi\right] \Psi^{-1} } & =i x \sigma_{3}-i \partial_{t} B_{+-} \sigma_{3}-\partial_{t} B_{++} \sigma_{2}
\end{aligned}
$$


and matrix $\Psi$ is indeed a matrix solution of a system similar to (1.13). The compatibility conditions for (2.10) are, of course, just Eqs. (1.14).

In all the above considerations the explicit form (1.8) of function $\vartheta$ was not used. It was essential that it possesses properties (1.16). It means that the Riemann problem may be used to construct solutions of Eqs. (1.14) depending on a functional parameter. The choice (1.7) for function $\vartheta$ leads to solutions $B_{++}, B_{+-}$which were defined earlier in (1.9).

To prove this last statement one analyzes the system of singular integral equations equivalent to the MRP. In the matrix form, these equations for function $\chi(\lambda)(2.1),(2.2)$ are written as

$$
\chi^{+}(\lambda)=I+\frac{1}{2 \pi i} \int_{-\infty}^{\infty} \frac{\chi^{+}(\mu)(I-G(\mu))}{\mu-\lambda-i 0} d \mu .
$$

The special $\operatorname{det}(I-G(\lambda))=0$ of matrix $G(2.3)$ allows us to reduce (2.13) to two scalar equations. Putting

$$
\hat{\chi}(\lambda)=\chi^{+}(\lambda) E(\lambda) ; \quad E(\lambda)=\left(\begin{array}{ll}
1 & e_{+}(\lambda) \\
0 & e_{-}(\lambda)
\end{array}\right)(\operatorname{Im} \lambda=0),
$$

and rewriting (2.11) for $\hat{\chi}$ one obtains two scalar integral equations for functions $\hat{\chi}_{12}, \hat{\chi}_{22}$,

$$
\begin{gathered}
\hat{\chi}_{l 2}(\lambda)-\gamma \int_{-\infty}^{\infty} K(\lambda, \mu) \hat{\chi}_{l 2}(\mu) d \mu=e_{l}(\lambda), \\
\left(l=1,2 ; e_{1} \equiv e_{+}, e_{2} \equiv e_{-}\right),
\end{gathered}
$$

where $K(\lambda, \mu)$ is just the nonsingular kernel (1.6) entering Eqs. (1.10) for functions $f_{ \pm}$. So

$$
\hat{\chi}_{12}(\lambda)=f_{+}(\lambda) ; \quad \hat{\chi}_{22}(\lambda)=f_{-}(\lambda),
$$

and the reduction of the system (2.11) to system (1.10) is realized.

The remaining two equations of the system (2.11) (rewritten for $\hat{\chi}$ ) are expressions of functions $\hat{\chi}_{l 1}=\chi_{l 1}(l=1,2)$ in terms of $\hat{\chi}_{l 2}$. Due to (2.14) they are written as

$$
\begin{gathered}
\chi_{l 1}(\lambda)=\sigma_{l 1}-\frac{\gamma}{2 i} \int_{-\infty}^{\infty} \frac{f_{l}(\mu) e_{-}(\mu)}{\mu-\lambda} d \mu(\operatorname{Im} \lambda>0) \\
\left(l=1,2 ; f_{1} \equiv f_{+} ; f_{2} \equiv f_{-}\right)
\end{gathered}
$$

Expanding now in $(1 / \lambda)$ one has for $\Psi_{1}$ in $(2.5)$,

$$
\left(\Psi_{1}\right)_{l 1}=\frac{\gamma}{2 i} \int_{-\infty}^{\infty} f_{l}(\mu) e_{-}(\mu) d \mu
$$

so that functions $B_{++}, B_{+-}$in (2.7) are indeed the same as those defined in (1.9).

We conclude the discussion of the Riemann problem with the following two remarks.

Remark 1. The equivalence of systems (1.10) and (2.11) established above allows us to interpret (1.10) as a GLM equation for nonlinear system (1.14). In turn, this 
gives the possibility to consider the Fredholm determinant (1.5) as a $\tau$-function for the system. This question will be discussed in more detail in a separate paper.

Remark 2. There exists an interesting relation of the objects discussed above to the spectral theory of the Dirac operator entering the $L-M$ pair (1.13). To demonstrate this, let us introduce scalar functions $\alpha(\lambda), \beta(\lambda)$ as (we suppose that (2.4) is valid)

$$
\left.\begin{array}{l}
\alpha(\lambda) \\
\beta(\lambda)
\end{array}\right\}=\exp \left\{-\frac{1}{2 \pi i} \int_{-\infty}^{\infty} \frac{d \mu}{\mu-\lambda} \ln g_{\alpha(\beta)}(\mu)\right\} .
$$

Functions $g_{\alpha}, g_{\beta}$ (depending also on $t, \gamma$ ) are

$$
g_{\alpha}(\lambda)=s_{0}(\lambda) / s_{1}(\lambda) ; \quad g_{\beta}(\lambda)=s_{0}(\lambda) / s_{-1}(\lambda)
$$

with functions $s_{0}, s_{1}, s_{-1}$ given as

$$
s_{p}(\lambda)=1-p \pi \gamma+\exp \left\{\mu^{2}-t\right\}(p=0,1,-1) .
$$

It is to be noted that functions $\alpha(\lambda), \beta(\lambda)$ are holomorphic for $\operatorname{Im} \lambda>0$ and $\operatorname{Im} \lambda<0$, $\alpha(\infty)=\beta(\lambda)=1$, and for real $\lambda$ they satisfy relation

$$
\alpha^{-}(\lambda)=\alpha^{+}(\lambda) g_{\alpha}(\lambda) ; \quad \beta^{-}(\lambda)=\beta^{+}(\lambda) g_{\beta}(\lambda),
$$

being solutions of the corresponding scalar Riemann problems. They allow us to transform matrix $G(2.3)$ to a matrix with diagonal elements equal to 1 . Going to the matrix-valued function $\Phi$,

$$
\Phi(\lambda)=\chi(\lambda)\left(\begin{array}{cc}
\beta(\lambda) & 0 \\
0 & \alpha(\lambda)
\end{array}\right)
$$

one changes $(2.2)$ to

$$
\begin{aligned}
\Phi^{-}(\lambda) & =\Phi^{+}(\lambda) G_{\Phi}(\lambda), \\
G_{\Phi}(\lambda) & =\left(\begin{array}{cc}
1, & b^{*}(\lambda) \exp \{2 i \lambda x\} \\
-b(\lambda) \exp \{-2 i \lambda x\}, & 1
\end{array}\right)
\end{aligned}
$$

with

$$
b(\lambda)=-\pi \gamma \beta^{-}(\lambda) \vartheta(\lambda) / \alpha^{+}(\lambda)
$$

obvious symmetry properties,

$$
\begin{aligned}
& \alpha^{*}\left(\lambda^{*}\right)=1 / \alpha(\lambda)=\alpha(-\lambda) \\
& \beta^{*}\left(\lambda^{*}\right)=1 / \beta(\lambda)=\beta(-\lambda)
\end{aligned}
$$

are taken into account). Comparing now matrix $G_{\Phi}$ with the conjugating matrix for the Dirac operator $L$ (see, e.g. [2]),

$$
L=-i \sigma_{3} \frac{d}{d x}+\left(\begin{array}{cc}
0 & u \\
u^{*} & 0
\end{array}\right)
$$

one comes to the conclusion that the finding function $B_{++}(x, t)$ is equivalent to 
the reconstructing potential $u$ for a given coefficient $b(\lambda)\left(u=i B_{++}\right)$. The fact that the potential appears to be pure imaginary is connected to properties (2.24).

\section{Generating Functional for Density Correlators $(\gamma<1 / \pi)$}

Here the asymptotics at $x \rightarrow \infty$ of correlator (1.2) for $-\infty<\alpha<0$ is calculated (the case $\alpha=-\infty$ is considered in the next section). The correlator we are interested in now is the Fredholm determinant $\Delta(1.5)$; due to (1.11) its logarithm $\sigma$ can be expressed as

$$
\sigma=-\int_{0}^{x} B_{+-}(\xi, t, \gamma) d \xi
$$

The partial differential equation (1.15) for $B_{++}$(remember that $B_{++}^{2}=\partial_{x} B_{+-}$) can be used to reproduce the complete asymptotic expansion, but one has to obtain first terms of the asymptotics before doing this.

The results obtained in Sect. 2 reduce the problem to calculating the asymptotics of matrix elements $\chi_{11}, \chi_{12}$ for the matrix $\chi(2.2)$. It is convenient to go to matrix $\Phi$ (2.2). Potentials $B$ can be extracted from the $(1 / \lambda)$-expansion $(2.5),(2.7)$ of $\chi(\lambda)$, or, equivalently, $\Phi(\lambda)$ :

$$
\begin{aligned}
& B_{+-}=2 i \lim _{\lambda \rightarrow \infty} \lambda\left(\Phi_{11}(\lambda)-\beta(\lambda)\right), \\
& B_{++}=-2 i \lim _{\lambda \rightarrow \infty} \lambda \Phi_{12}(\lambda),
\end{aligned}
$$

(functions $\alpha(\lambda)$ and $\beta(\lambda)$ which are often used below are defined in (2.17)). To find the asymptotics of $\Phi$ one begins with the system of integral equations (2.11) which is readily rewritten for functions as

$$
\begin{aligned}
& \Phi_{11}^{+}(\lambda)=1-\frac{\gamma}{2 i} \int_{-\infty}^{\infty} \frac{\Phi_{12}^{+}(\mu) \beta^{-}(\mu) \exp \{-2 i \mu x\}}{(\mu-\lambda-i 0) \alpha^{-}(\mu) s_{1}(\mu)} d \mu, \\
& \Phi_{12}^{+}(\lambda)=\frac{\gamma}{2 i} \int_{-\infty}^{\infty} \frac{\Phi_{11}^{+}(\mu) \alpha^{+}(\mu) \exp \{2 i \mu x\}}{(\mu-\lambda-i 0) \beta^{+}(\mu) s_{1}(\mu)} d \mu,
\end{aligned}
$$

where functions $s_{p}(p=0, \pm 1)$ are given by (2.19). For functions $\Phi$ the following estimates are valid:

$$
\begin{array}{r}
\left|\Phi_{11}(\lambda, x, t)-1\right| \leqq C / \lambda, \\
\left|\Phi_{12}(\lambda, x, t)\right| \leqq D / \lambda,
\end{array}
$$

where $\operatorname{Im} \lambda \geqq 0, t \leqq t_{0}, x \geqq x_{0}$ and constants $C, D$ depend on $x_{0}, t_{0}$ only (for $\gamma$ fixed). These estimates can be obtained directly from (3.3) and (3.4) (one can also make use of Remark 2 of Sect. 2 to interpret $\Phi_{i k}$ as the Jost solutions for the Dirac operator $L$ which permits to use standard facts [2] of the scattering theory for this operator).

Let us now take into account that function $\Phi_{11}(\mu) \alpha(\mu) / \beta(\mu)$ is analytical in the upper half-plane and also satisfies estimate (3.5) (remember that $\Phi_{11}(\infty)=\alpha(\infty)=$ 
$\beta(\infty)=1)$. Hence the integration contour in (3.4) can be shifted into the upper half-plane and the integral can be represented as a sum of residues at first-order poles at points $\lambda_{k},-\lambda_{k}^{*}$ (which are zeros of function $s_{1}(\lambda)$ in the upper half-plane):

$$
\lambda_{K}=[\ln (1-\pi \gamma)+t+(2 k+1) i \pi]^{1 / 2} ; \quad \operatorname{Im} \lambda_{K}>0, \quad \operatorname{Re} \lambda_{K}>0 .
$$

Thus the following representation is obtained for function $\Phi_{12}$ :

$$
\Phi_{12}^{+}(\lambda)=\frac{\pi \gamma \Phi_{11}^{+}(\lambda) \alpha^{+}(\lambda) \exp \{2 i \lambda x\}}{s_{1}(\lambda) \beta^{+}(\lambda)}+S(\lambda)
$$

where $S(\lambda)$ is given as a series,

$$
S(\lambda)=\sum_{k=0}^{\infty}\left(S\left(\lambda_{K}, \lambda\right)+S\left(-\lambda_{K}^{*}, \lambda\right)\right)
$$

and

$$
S\left(\lambda_{K}, \lambda\right)=\frac{\pi \gamma \Phi_{11}\left(\lambda_{K}\right) \alpha\left(\lambda_{K}\right) \exp \left\{2 i \lambda_{K} x\right\}}{2(\pi \gamma-1) \lambda_{K}\left(\lambda_{K}-\lambda\right) \beta\left(\lambda_{K}\right)}
$$

series (3.9) is uniformly convergent for $\lambda \in \mathbb{R}, x \geqq x_{0}$ and $t_{1} \leqq t \leqq t_{0}$ (where $t_{1}$ is an arbitrary but finite constant). This series is also asymptotical at $x \rightarrow+\infty$ uniformly for $\lambda \in \mathbb{R}, t_{1} \leqq t \leqq t_{0}$ (from now on it is supposed that condition (2.4) is fulfilled). $\Phi_{11}(\lambda)$

Putting expression (3.8) into (3.3) one comes to the representation for function

$$
\Phi_{11}^{+}(\lambda)=1-\frac{\pi \gamma^{2}}{2 i} \int_{-\infty}^{\infty} \frac{\Phi_{11}^{+}(\mu) d \mu}{S_{1}(\mu) S_{-1}(\mu)(\mu-\lambda-i 0)}-\frac{1}{2 \pi i} \int_{-\infty}^{\infty} \frac{R(\mu) d \mu}{(\mu-\lambda-i 0)}
$$

with

$$
R(\mu)=\frac{\pi \gamma \beta^{-}(\mu) \exp \{-2 i \mu x\}}{\alpha^{-}(\mu) S_{1}(\mu)} S(\mu) .
$$

Equation (3.11) may be regarded as a singular integral equation for the inhomogeneous scalar RP:

$$
\tilde{\Phi}_{11}^{-}(\lambda)=\Phi_{11}^{+}(\lambda) g(\lambda)+R(\lambda)
$$

where due to (2.20), function $g(\lambda)$ is given as

$$
g(\lambda)=\alpha^{-}(\lambda) \beta^{-}(\lambda) / \alpha^{+}(\lambda) \beta^{+}(\lambda),
$$

which solves the problem. Using the fact that $\Phi_{11}(\infty)=1$, one has

$$
\Phi_{11}(\lambda)=\alpha(\lambda) \beta(\lambda)-\frac{\alpha(\lambda) \beta(\lambda)}{2 \pi i} \int_{-\infty}^{\infty} \frac{R(\mu) d \mu}{\alpha^{-}(\mu) \beta^{-}(\mu)(\mu-\lambda)} \quad(\operatorname{Im} \lambda \geqq 0),
$$

or in a more explicit form (see (3.12), (3.9), (3.10)),

$$
\begin{gathered}
\Phi_{11}(\lambda)=\alpha(\lambda) \beta(\lambda)-\frac{\alpha(\lambda) \beta(\lambda)}{2 \pi i} \sum_{k=0}^{\infty}\left[A\left(\lambda_{K}, \lambda\right) \Phi_{11}\left(\lambda_{K}\right)\right. \\
\left.+A\left(-\lambda_{K}^{*}, \lambda\right) \Phi_{11}\left(-\lambda_{K}^{*}\right)\right] \quad(\operatorname{Im} \lambda \geqq 0),
\end{gathered}
$$


where

$$
\begin{aligned}
A\left(\lambda_{K}, \lambda\right)= & \frac{\pi^{2} \gamma^{2}}{2(\pi \gamma-1) \lambda_{K}} \frac{\alpha\left(\lambda_{K}\right)}{\beta\left(\lambda_{K}\right)} \exp \left\{2 i \lambda_{K} x\right\} \\
& \cdot \int_{-\infty}^{\infty} \frac{\exp \{-2 i \mu x\} d \mu}{\left(\alpha^{-}(\mu)\right)^{2} s_{1}(\mu)\left(\lambda_{K}-\mu\right)(\mu-\lambda)} .
\end{aligned}
$$

This representation allows us, in particular, to obtain the first term of the asymptotics of $\Phi_{11}$ at $x \rightarrow+\infty$ (see also Remark 1 in this section). Due to the possibility of shifting the integration contour in the integral in (3.15) into the lower half-plane and to the estimate (3.5), these integrals can be estimated by $C\left|\exp \left\{2 i \lambda_{0} x\right\} /\left(\lambda+\lambda_{0}\right)\right|$. Hence one has

$$
\Phi_{11}(\lambda)=\alpha(\lambda) \beta(\lambda)+\mathcal{O}\left(\exp \left\{-4 r_{0} x \sin \varphi_{0}\right\}\right), \quad(x \rightarrow+\infty),
$$

where $r_{0}=\left|\lambda_{0}\right|, \varphi_{0}=\arg \lambda_{0}$ (see (3.7)).

From (3.4) one also obtains

$$
\begin{aligned}
\Phi_{12}(\lambda)= & \frac{\gamma}{2 i} \int_{-\infty}^{\infty} \frac{\left(\alpha^{+}(\mu)\right)^{2} \exp \{2 i \mu x\} d \mu}{s_{1}(\mu)(\mu-\lambda)} \\
& +\mathcal{O}\left(\exp \left\{-4 r_{0} x \sin \varphi_{0}\right\}\right) \quad(x \rightarrow+\infty) .
\end{aligned}
$$

These estimates are uniform for $\operatorname{Im} \lambda \geqq 0, t_{1} \leqq t \leqq t_{0}$. Expanding in $(1 / \lambda)($ see (3.2)) one gets for potentials $B$,

$$
\begin{aligned}
B_{+-}= & \frac{1}{\pi} \int_{-\infty}^{\infty} d \lambda \ln \frac{1+\exp \left\{\lambda^{2}-t\right\}}{1-\pi \gamma+\exp \left\{\lambda^{2}-t\right\}} \\
& +\mathcal{O}\left(\exp \left\{-4 r_{0} x \sin \varphi_{0}\right\}\right) \quad(x \rightarrow+\infty), \\
B_{++}= & \gamma \int_{-\infty}^{\infty} \frac{\left(\alpha^{+}(\lambda)\right)^{2} \exp \{2 i \lambda x\}}{\left(1-\pi \gamma+\exp \left\{\lambda^{2}-t\right\}\right)} d \lambda \\
& +\mathcal{O}\left(\exp \left\{-4 r_{0} x \sin \varphi_{0}\right\}\right) \quad(x \rightarrow+\infty)
\end{aligned}
$$

(we substituted functions $s_{p}$ (2.19) in the explicit form). Shifting the integration contour in the last formula to the upper half-plane one obtains a more exact estimate for $B_{++}$,

$$
\begin{aligned}
B_{++}= & \frac{2 \pi \gamma}{1-\pi \gamma} \sum_{k=0}^{K} \frac{\left|\alpha^{+}\left(\lambda_{K}\right)\right|^{2}}{r_{K}} \exp \left\{-2 x r_{k} \sin \varphi_{K}\right\} \\
& \cdot \sin \left\{2 x r_{K} \cos \varphi_{k}+2 \arg \alpha\left(\lambda_{K}\right)-\varphi_{K}\right\}+\mathcal{O}\left(\exp \left\{-4 x r_{0} \sin \varphi_{0}\right\}\right),
\end{aligned}
$$

where $r_{K}=\left|\lambda_{K}\right|, \varphi_{K}=\arg \lambda_{K}$ and integer $K=K(t, \gamma)$ has to be defined from conditions

$$
\left\{\begin{array}{c}
r_{K} \sin \varphi_{K} \equiv \operatorname{Im} \lambda_{K}<2 \operatorname{Im} \lambda_{0} \equiv 2 r_{0} \sin \varphi_{0} \\
r_{K+1} \sin \varphi_{K+1}>2 r_{0} \sin \varphi_{0}
\end{array} .\right.
$$

It should be noted that, using Eq. (1.14), $\partial_{x} B_{+-}=B_{++}^{2}$, one can obtain the 
next terms of the asymptotics of function $B_{+}$- by means of (3.20):

where

$$
\begin{aligned}
B_{+-}= & a-b^{2}\left(8 r_{0}\right)^{-1} \exp \left\{-4 x r_{0} \sin \varphi_{0}\right\} \\
& \cdot\left[\left(\sin \varphi_{0}\right)^{-1}+\sin \left\{4 x r_{0} \cos \varphi_{0}+2 \theta_{0}-\varphi_{0}\right\}\right] \\
& +\mathcal{O}\left(\exp \left\{-4 x r_{0} \sin \varphi_{0}\right\}\right), \quad x \rightarrow+\infty,
\end{aligned}
$$

$$
\begin{aligned}
& a=\frac{1}{\pi} \int_{-\infty}^{\infty} d \lambda \ln \frac{1+\exp \left\{\lambda^{2}-t\right\}}{1-\pi \gamma+\exp \left\{\lambda^{2}-t\right\}}=a(t), \\
& b=\frac{2 \pi \gamma}{1-\pi \gamma} \frac{\left|\alpha\left(\lambda_{0}\right)\right|^{2}}{r_{0}} ; \quad \theta_{0}=2 \arg \alpha\left(\lambda_{0}\right)-\varphi_{0} .
\end{aligned}
$$

So we have obtained the first terms of the asymptotics of potentials. Apply now these results to get the asymptotics of function $\sigma=\ln \Delta$, which is just the logarithm of correlator (1.2). Using representation (3.1) and Eqs. (1.12), (3.20) and (3.22), one obtains:

$$
\begin{aligned}
\sigma= & -a(t) x+\frac{1}{2} \int_{-\infty}^{t} d \tau\left(\frac{d a(\tau)}{d \tau}\right)+\kappa-b^{2}\left(32 r_{0}^{2}\right)^{-1} \exp \left\{-4 x r_{0} \sin \varphi_{0}\right\} \\
& \cdot\left[\left(\sin \varphi_{0}\right)^{-2}+\cos \left\{4 x r_{0} \cos \varphi_{0}+2 \theta_{0}-2 \varphi_{0}\right\}\right] \\
& +\mathcal{O}\left(\exp \left\{-4 x r_{0} \sin \varphi_{0}\right\}\right) \quad(x \rightarrow+\infty) .
\end{aligned}
$$

Constant $\kappa$ here does not depend on $x, t$, being a function of $\gamma$ only: $\kappa=\kappa(\gamma)$. Its exact value is unknown to us. Asymptotics (3.24) is the main result of this section. It is uniform in $t$ at any (but finite) interval $t_{1} \leqq t \leqq t_{0}$. Asymptotics of correlator (1.2) is readily obtained as

$$
\langle\exp \{\alpha Q(z)\}\rangle_{T}=\exp \{\sigma(x / 2, t, \gamma)\},
$$

where one should put $x=z \sqrt{T}, t=h / T, \gamma=(1-\exp \{\alpha\}) / \pi$. Before going further we would like to make the following two remarks.

Remark 1. Equations (3.18) and (3.20) give the main terms of asymptotics of potentials $B_{++}, B_{+-}$. Next terms and, in principle, complete asymptotic expansions can be obtained from the nonlinear differential equation (1.15) for function $B_{++}$, if one substitutes the following ansatz into it:

$$
\begin{aligned}
B_{++}= & \sum A_{K} e^{-2 x r_{K} \sin \varphi_{K}} \sin \left\{2 x r_{K} \cos \varphi_{K}+\theta_{K}\right\}+\sum A_{k l}^{ \pm} e^{-2 x\left(r_{K} \sin \varphi_{K}+r_{l} \sin \varphi_{l}\right)} \\
& \cdot \sin \left\{2\left(r_{K} \sin \varphi_{K} \pm r_{l} \sin \varphi_{l}\right) x+\theta_{k l}\right\}+\cdots .
\end{aligned}
$$

We use this approach in the next section in the more simple case $\gamma=1 / \pi$.

Another way to obtain asymptotic expansions is to use representation (3.14). Putting there $\lambda=\lambda_{K}, \lambda=-\lambda_{K}^{*}$, one obtains an infinite dimensional linear system for coefficients $\Phi\left(\lambda_{K}\right), \Phi\left(-\lambda_{K}^{*}\right)$ which can be used to construct asymptotic expansions.

Remark 2. It was already mentioned that from the point of view of the scattering theory for the Dirac operator $L(2.25)$, potential $B_{++}$corresponds to the reflection 
coefficient $b(\lambda)(2.23)$. It is not difficult to restore the transition coefficient $a(\lambda)$,

$$
a(\lambda)=\exp \left\{\frac{1}{2 \pi i} \int_{-\infty}^{\infty} \frac{\ln \left(1+|b(\mu)|^{2}\right)}{\mu-\lambda-i 0} d \mu\right\}=\left(\alpha^{+}(\lambda) \beta^{+}(\lambda)\right)^{-1} .
$$

For the "scattering amplitude" $r(\lambda)$ one then has

$$
r(\lambda) \equiv b^{*}(\lambda) / a(\lambda)=-\pi \gamma\left(\alpha^{+}(\lambda)\right)^{2}\left(1-\pi \gamma+\exp \left\{\lambda^{2}-t\right\}\right)^{-1},
$$

so formula (3.19) obtained for $B_{++}$can be interpreted as a well-known Born approximation.

\section{Emptiness Formation Probability $(\gamma=1 / \pi)$}

The emptiness formation probability (1.3), being a special case at $\alpha=-\infty$ of correlator (1.2), is the Fredholm determinant $\Delta$ with $\gamma=1 / \pi$. Below the complete asymptotic expansion of the logarithm of this correlator, $\sigma(x, t, \gamma=1 / \pi)=$ $\ln \Delta(x, t, \gamma=1 / \pi)$ is obtained. As was already explained at the beginning of Sect. 3, the problem is reduced to the asymptotic analysis of the system of singular integral equations (3.3) and (3.4). The method of the analysis is, in principle, the same, but estimates are essentially different. In particular, they are now uniform in the interval $t \leqq t_{0}$ including $t=-\infty$. For example, representation (3.8) is now changed for

$$
\Phi_{12}^{+}(\lambda)=\frac{\pi \gamma \Phi_{11}^{+}(\lambda) \alpha^{+}(\lambda) \exp \left\{2 i \lambda x-\lambda^{2}+t\right\}}{\beta^{+}(\lambda)}+\exp \left\{-x^{2}+t\right\} R(\lambda),
$$

where $R(\lambda)$ is a function continuous at $\lambda \in \mathbb{R}$, holomorphic for $\operatorname{Im} \lambda<0$ and satisfying the estimate $|R(\lambda)|<C / \sqrt{|\lambda|^{2}+x^{2}}$ uniformly for $\operatorname{Im} \lambda \leqq 0, t \leqq t_{0}$ and $x \geqq x_{0}$. Repeating the corresponding considerations of Sect. 2 (but taking into account (4.1)) one obtains for $\Phi_{11}$ instead of (3.13),

$$
\Phi_{11}(\lambda)=\alpha(\lambda) \beta(\lambda)-\frac{\alpha(\lambda) \beta(\lambda)}{2 \pi i} \exp \left\{-2 x^{2}+t\right\} \int_{-\infty}^{\infty} \frac{R(\mu-i x) \exp \left\{-\mu^{2}\right\}}{\alpha^{2}(\mu-i x)(\mu-\lambda-i x)} d \mu,
$$

so that

$$
\Phi_{11}(\lambda)=\alpha(\lambda) \beta(\lambda)+\exp \left\{-2 x^{2}+t\right\} L(\lambda),
$$

where function $L(\lambda)$ is continuous for $\lambda \in \mathbb{R}$, holomorphic at $\operatorname{Im} \lambda \geqq 0$ and satisfies the estimate, $|L(\lambda)|<C / x \sqrt{|\lambda|^{2}+x^{2}}$ uniformly for $\operatorname{Im} \lambda \leqq 0, t \leqq t_{0}, x \geqq x_{0}$. The asymptotic representation of $\Phi_{12}$ is obtained from (3.4) and (4.3). At $\operatorname{Im} \lambda>0$,

$$
\Phi_{12}(\lambda)=\frac{1}{2 \pi i} \int_{-\infty}^{\infty} \frac{d \mu\left(\alpha^{+}(\mu)\right)^{2} \exp \left\{2 i \mu x+t-\mu^{2}\right\}}{(\mu-\lambda)}+\exp \left\{-3 x^{2}+3 t\right\} M(\lambda),
$$

where $|M(\lambda)|<C / x^{2} \sqrt{|\lambda|^{2}+x^{2}}$.

Thus one has, due to (3.2), the following asymptotics for potentials $B$,

$$
\begin{aligned}
& B_{+-}(x, t)=\frac{1}{\pi} \int_{-\infty}^{\infty} d \lambda \ln \left(1+e^{t-\lambda^{2}}\right)+\mathcal{O}\left(\exp \left\{-2 x^{2}+2 t\right\} / x\right) \quad(x \rightarrow+\infty), \\
& B_{++}(x, t)=\frac{1}{\pi} \int_{-\infty}^{\infty} d \lambda\left(\alpha^{+}(\lambda)\right)^{2} \exp \left\{2 i \lambda x+t-\lambda^{2}\right\}+\mathcal{O}\left(\exp \left\{-3 x^{2}+3 t\right\} / x^{2}\right) .
\end{aligned}
$$


These estimates are uniform for $t \leqq t_{0}$ including $t=-\infty$ (which differs from (3.18), (3.19) in this aspect).

Construct now the complete asymptotical expansions for $B_{++}, B_{+-}$, using the partial differential equation for $B_{++}(1.15)$. One begins with the representation of the integral in (4.6) as

$$
\begin{aligned}
& \frac{1}{\pi} \int_{-\infty}^{\infty} d \lambda\left(\alpha^{+}(\lambda)\right)^{2} \exp \left\{2 i \lambda x+t-\lambda^{2}\right\} \\
& \quad=\exp \left\{-x^{2}+t\right\}\left(\frac{1}{\sqrt{\pi}}+\frac{1}{\pi} \sum_{k, l=0}^{\infty} \frac{(-1)^{l} \alpha_{K}(2 l+k) !}{x^{2 l+k+1} i^{K+1} k !(2 l) !} \Gamma\left(l+\frac{1}{2}\right)\right)
\end{aligned}
$$

where $\alpha_{K}$ are introduced by means of the expansion of function $\alpha(\lambda)(2.17)$ (it is to be remembered that $\gamma=1 / \pi$ ) as

$$
\alpha^{2}(\lambda)=1+\sum_{k=0}^{\infty}\left(\alpha_{K} / \lambda^{k+1}\right), \quad \lambda \rightarrow \infty, \quad \operatorname{Im} \lambda>0 .
$$

Due to the explicit form of $\alpha(\lambda)$, all $\alpha_{K}$ 's can be calculated from the identity

$$
\begin{gathered}
1+\sum_{K=0}^{\infty}\left(\alpha_{K} / \lambda^{k+1}\right) \equiv \exp \left\{-i \sum_{K=0}^{\infty} c_{k} / \lambda^{2 k+1}\right\}, \\
c_{k}=\frac{1}{\pi} \int_{-\infty}^{\infty} \lambda^{2 k} \ln \left(1+\exp \left\{t-\lambda^{2}\right\}\right) d \lambda .
\end{gathered}
$$

Thus one obtains

$$
\alpha_{0}=-i c_{0}, \alpha_{1}=-c_{0}^{2} / 2, \alpha_{2}=-i c_{1}+\left(i c_{0}^{3} / 6\right), \alpha_{3}=-c_{0} c_{1}+\left(c_{0}^{4} / 4 !\right), \text { etc } .
$$

Returning now to the asymptotics (4.6) for $B_{++}$, one can write

$$
B_{++}(x, t)=\exp \left\{-x^{2}+t\right\} \sum_{n=0}^{\infty}\left(a_{n} / x^{n}\right)+\mathcal{O}\left(\exp \left\{-3 x^{2}+3 t\right\} / x^{2}\right) \quad(x \rightarrow+\infty),
$$

where coefficients $a_{n}$ (functions of $t$ ) are expressed in terms of $\alpha_{K}$ :

$$
\begin{aligned}
& a_{0}=1 / \sqrt{\pi}, a_{1}=\alpha_{0} / i \sqrt{\pi}, a_{2}=\alpha_{1} / i^{2} \sqrt{\pi} ; \\
& a_{n}=\frac{1}{\pi} \sum_{\substack{k, l=0, \ldots \infty \\
k+2 l=n-1}} \frac{(-i)^{l} \alpha_{k}(2 l+k) !}{i^{k+1} k !(2 l) !} \Gamma\left(l+\frac{1}{2}\right)
\end{aligned}
$$

(it is to note that the sum here are taken over integers $k, l$ ).

Turn now to the nonlinear partial differential equation (1.15) for $B_{++}(x, t)$. Estimate (4.9) suggests the following ansatz:

$$
\begin{aligned}
B_{++}(x, t)= & \exp \left\{-x^{2}+t\right\}\left(a_{0}+\frac{a_{1}}{x}+\frac{a_{2}}{x^{2}}+\frac{a_{3}}{x^{3}}+\cdots\right) \\
& +\exp \left\{-3 x^{2}\right\}\left(\frac{b_{2}}{x^{2}}+\frac{b_{3}}{x^{3}}+\frac{b_{4}}{x^{4}}+\cdots\right)
\end{aligned}
$$




$$
\begin{aligned}
& +\exp \left\{-5 x^{2}\right\}\left(\frac{d_{4}}{x^{4}}+\frac{d_{5}}{x^{5}}+\cdots\right)+\cdots \\
& +\exp \left\{-(2 N+1) x^{2}\right\}\left(\frac{d_{2 N}^{(N)}}{x^{2 N}}+\frac{d_{2 N+1}^{(N)}}{x^{2 N+1}}+\cdots\right)+\cdots
\end{aligned}
$$

Quantities $a_{j}, b_{j}, d_{j} \cdots d_{j}^{(N)}, \ldots$ are functions of $t$ which should be (except for known $a_{j}(4.10)$ ) determined. Putting formal series (4.11) into Eq. (1.15) and taking into account (4.5) one obtains for functions $b, d, d^{(N)}$ the linear ordinary differential equations in $t$, e.g. for $b_{2}, b_{3}, b_{4}$, one has

$$
\begin{aligned}
& \partial_{t} b_{2}-(1 / 3) b_{2}=\left(1 / 3 \pi^{3 / 2}\right) e^{3 t} \\
& \partial_{t} b_{3}-(1 / 3) b_{3}=\left(3 / 16 \pi^{3 / 2}\right) e^{t / 3} \partial_{t}\left(e^{8 t / 3} c_{0}\right) \\
& \partial_{t} d_{4}-(1 / 5) b_{4}=\left(1 / 80 \pi^{5 / 2}\right) e^{5 t}
\end{aligned}
$$

The solutions of these equations are defined up to solutions of the corresponding homogeneous equations, these being

$$
\begin{aligned}
& B_{2}(t)=\text { const } \cdot \exp \{t / 3\}, B_{3}(t)=\text { const } \cdot \exp \{t / 3\} \\
& D_{4}(t)=\text { const } \cdot \exp \{t / 5\}
\end{aligned}
$$

All the constants here should be, however, equal to zero as functions $b(t), d(t) \ldots$ should not contain fractional powers of $\exp \{t\}$ in the expansion at $t \rightarrow-\infty$. This is the direct consequence of the uniformity for $t \leqq t_{0}$ of estimate (4.6) (see also Sect. 6 of paper [1] where the explicit expansion at $t \rightarrow-\infty$ is constructed). Thus one has from (4.12),

$$
\begin{aligned}
& b_{2}(t)=-\left(1 / 16 \pi^{3 / 2}\right) \exp \{3 t\}, \\
& b_{3}(t)=\left(3 / 16 \pi^{3 / 2}\right) \exp \{3 t\} c_{0}(t), \\
& d_{4}(t)=\left(1 / 384 \pi^{5 / 2}\right) \exp \{5 t\} .
\end{aligned}
$$

Arguments of this kind are valid also for any higher terms in the expansion (4.11). It should be emphasized, however, that it is essential that coefficients $a_{j}$ are already defined independently. The reason is that a solution of the corresponding homogeneous equation is $\exp \{t\}$ and arguments about the presence of only entire powers of $\exp \{t\}$ give nothing in this case.

Thus one comes to the following asymptotic expansion of potential $B_{++}$:

$$
\begin{aligned}
B_{++}(x, t)= & \exp \left\{-x^{2}+t\right\} \sum_{n=0}^{\infty} \frac{a_{n}}{x^{n}} \\
& +\exp \left\{-3 x^{2}+3 t\right\}\left(-\frac{1}{16 \pi^{3 / 2}} \frac{1}{x^{2}}+\frac{3}{16 \pi^{3 / 2}} \frac{c_{0}(t)}{x^{3}}+\cdots\right) \\
& +\exp \left\{-5 x^{2}+5 t\right\}\left(\frac{1}{384 \pi^{5 / 2}} \frac{1}{x^{4}}+\cdots\right)+\cdots \quad(x \rightarrow+\infty),
\end{aligned}
$$

where functions $a_{n}=a_{n}(t)$ are given by (4.10) and $c_{0}(t)$ is defined in (4.7). Using 
now (4.5) and equation $\partial_{x} B_{+-}=B_{++}^{2}(1.14)$ one has for potential $B_{+--}$,

$$
\begin{aligned}
B_{+-}(x, t)= & c_{0}(t)+\exp \left\{-2 x^{2}+2 t\right\} \sum_{n=1}^{\infty} \frac{A_{n}}{x^{n}} \\
& +\exp \left\{-4 x^{2}+4 t\right\}\left(\frac{1}{2^{7} \pi^{2}} \frac{1}{x^{3}}+\cdots\right)+\cdots(x \rightarrow+\infty),
\end{aligned}
$$

where functions $A_{n}=A_{n}(t)$ are calculated from the identity

$$
\begin{gathered}
\exp \left\{-2 x^{2}+2 t\right\} \sum_{n=1}^{\infty}\left(A_{n} / x^{n}\right)=-\int_{x}^{\infty} d y \exp \left\{-2 y^{2}+2 t\right\} \sum \frac{a_{n} a_{m}}{y_{n+m}}, \\
A_{1}=-\frac{1}{4 \pi} ; A_{2}=-\frac{a_{1}}{2 \sqrt{\pi}} ; A_{3}=\frac{1}{16 \pi}-\frac{a_{1}^{2}}{4}-\frac{a_{2}}{2 \sqrt{\pi}} .
\end{gathered}
$$

Equation (3.1) may now be used to derive the asymptotics of function $\sigma(x, t)=\ln \Delta(x, t)$ :

$$
\begin{aligned}
\sigma(x, t, \gamma=1 / \pi)= & -x c_{0}(t)+\frac{1}{2} \int_{-\infty}^{t}\left(\partial_{t} c_{0}\right)^{2} d t \\
& +\exp \left\{-2 x^{2}+2 t\right\}\left[\frac{A_{1}}{4 x^{2}}+\frac{A_{2}}{4 x^{3}}+\left(\frac{A_{1}}{2}-A_{3}\right) \frac{1}{4 x^{4}}+\cdots\right] \\
& +\exp \left\{-4 x^{2}+4 t\right\}\left[\frac{1}{2^{10} \pi^{2} x^{4}}+\cdots\right]+\cdots \quad(x \rightarrow+\infty) .
\end{aligned}
$$

It should be noted that at $\gamma=1 / \pi$ there is no problem with the integration constant $\kappa$ as it was in the case $\gamma<1 / \pi(3.24)$. Due to the uniformity of all the estimates in this section for $t \leqq t_{0}$ one can take the limit $t \rightarrow-\infty$ in (4.16) and use the fact that in this limit $\sigma=0$ (1.11).

Remember that the emptiness formation probability (1.3) is given as

$$
P(z, h, T)=\exp \{\sigma(x / 2, t, \gamma=1 / \pi\}, \quad x=z \sqrt{T}, t=h / T,
$$

so one easily the long-distance expansion by virtue of (4.16). We write explicitly only the most interesting first term:

$$
\begin{aligned}
P(z, h, T) & =\exp \left\{\frac{1}{2} \int_{-\infty}^{h / T}\left(\partial_{\tau} c_{0}(\tau)\right)^{2} d \tau\right\} \times \exp \left\{-z \sqrt{T} c_{0}(h / T) / 2\right\}, \quad(z \rightarrow+\infty) \\
c_{0}(t) & =\frac{1}{\pi} \int_{-\infty}^{\infty} \ln \left(1+\exp \left\{t-\lambda^{2}\right\}\right) d \lambda
\end{aligned}
$$

\section{Two-Point Field Correlator $(\gamma=2 / \pi)$}

The asymptotics at $z \rightarrow+\infty$ of correlator $\left\langle\psi^{+}(z) \psi(-z)\right\rangle_{T}(1.1)$ is briefly discussed here (we hope to give the complete asymptotic expansion in a separate paper). Remember that correlator (1.1) is the product $(\sqrt{T} / 4) B_{+} \Delta$, where the potential $B_{++}$and the Fredholm determinant $\Delta$ should be taken at $\gamma=2 / \pi$. 
First consider the problem for the negative chemical potentials $h<0$ (i.e. for $t<0$ ). In this case (see (2.4a)) taking into account standard results [4] one concludes that the Riemann problem (2.2) is uniquely solvable for all $x$. So one can repeat the consideration of Sect. 3 to obtain the asymptotics. The only essential alteration is that now zeros $\lambda_{K},-\lambda_{K}^{*}$ of functions $s_{1}(\lambda)(2.19)$,

$$
s_{1}(\lambda)=\exp \left\{\lambda^{2}-t\right\}-1 \quad(\gamma=2 / \pi)
$$

are given, instead of (3.7), as

$$
\lambda_{K}=[t+2 i k \pi]^{1 / 2}
$$

so that $\lambda_{0}$ is now pure imaginary, $\lambda_{0}=-\lambda_{0}^{*}=i|t|^{1 / 2}$. Hence only one term with $k=0$ should be kept in series (3.9), (3.14). Correspondingly, the term with $k=0$ in expansion (3.20) for $B_{++}$should be taken with coefficient $1 / 2$, being equal to

$$
2|t|^{-1 / 2}\left(\alpha\left(\lambda_{0}\right)\right)^{2} \exp \left\{-2 x|t|^{1 / 2}\right\} \quad(\gamma=2 / \pi)
$$

(we take into account that $r_{0}=|t|^{1 / 2}, \varphi_{0}=\pi / 2$ and $\arg \alpha\left(\lambda_{0}\right)=0$ ). For $\alpha\left(\lambda_{0}\right)$ one obtains

$$
\alpha\left(\lambda_{0}\right)=\exp \left\{-\frac{|t|^{1 / 2}}{2 \pi} \int_{-\infty}^{\infty} \frac{d \mu}{\mu^{2}+|t|} \ln \frac{\exp \left\{\mu^{2}+|t|\right\}+1}{\exp \left\{\mu^{2}+|t|\right\}-1}\right\} .
$$

Other terms remain the same (of course, $\gamma=2 / \pi$ in all the formulae). The nondecreasing terms in the asymptotics of $\sigma=\ln \Delta$ (3.24) remain unchanged and one obtains for the asymptotics of the correlator $\left\langle\psi^{+}(z) \psi(-z)\right\rangle_{T}$,

$$
\begin{aligned}
\left\langle\psi^{+}(z) \psi(-z)\right\rangle_{T}= & \rho_{0} T|h|^{-1 / 2}\left(\alpha\left(\lambda_{0}\right)\right)^{2} \exp \left\{\frac{1}{2} \int_{-\infty}^{t} d \tau\left(\frac{d c(\tau)}{d \tau}\right)^{2}\right\} \\
& \cdot \exp \left\{-z\left(2|h|^{1 / 2}+T^{1 / 2} c(t)\right)\right\} ; z \rightarrow+\infty, t=\frac{h}{T}<0 .
\end{aligned}
$$

Here

$$
c(t)=\frac{1}{\pi} \int_{-\infty}^{\infty} d \lambda \ln \left(\frac{\exp \left\{\lambda^{2}-t\right\}+1}{\exp \left\{\lambda^{2}-t\right\}-1}\right)
$$

and $\rho_{0}$ is a constant.

Turn now to the case of positive chemical potentials $h>0$ (i.e. $t>0$ ), which is more complicated. The sufficient conditions [4] of the solvability of the Riemann problem are not fulfilled for real $\gamma>1 / \pi$. From a technical point of view, the difficulty is that zeros $\lambda_{0},-\lambda_{0}^{*}$ in (5.2) become real,

$$
\lambda_{0}=t^{1 / 2},-\lambda_{0}^{*}=-t^{1 / 2} \quad(t>0, \gamma=2 / \pi),
$$

so that estimates of Sect. 3 are no more valid. More exactly, the first term in the series analogous to that in (3.14) is now not small at $x \rightarrow+\infty$ and should be included into the main term of the asymptotics. To avoid the difficulty one regularizes the problem going to the complex parameter $\gamma: \gamma \rightarrow \gamma+i \varepsilon$ and then taking the limit $\varepsilon \rightarrow 0$. It appears that the answers do not depend on the sign of $\varepsilon$ (one can also make $t$ complex and then go to real $t$; the answers are the same). 
Starting from the system (3.3) and (3.4) of singular integral equations one derives the representation similar to (3.14) and uses it to calculate the asymptotics as explained in Remark 1 of Sect. 3. Not going into more detail, let us give the answer for the main term of the asymptotics of correlator (1.1) thus obtained (see also [5]):

$$
\begin{aligned}
\left\langle\psi^{+}(z) \psi(-z)\right\rangle_{T}= & \rho_{\infty} T^{1 / 2} \pi^{-1 / 2} \exp \left\{-\frac{1}{2} \int_{t}^{\infty} d \tau\left(\frac{d c(\tau)}{d \tau}\right)^{2}\right\} \\
& \cdot \exp \left\{-z T^{1 / 2} c(t)\right\}, z \rightarrow+\infty, t=\frac{h}{T}<0
\end{aligned}
$$

Here

$$
c(t)=\frac{1}{\pi} \int_{-\infty}^{\infty} d \lambda \ln \left|\frac{\exp \left\{\lambda^{2}-t\right\}+1}{\exp \left\{\lambda^{2}-t\right\}-1}\right|
$$

and constant $\rho_{\infty}$ is the same as in paper [6] where the long-distance asymptotics of the correlator at zero temperature was calculated: $\rho_{\infty}=\pi e^{1 / 2} 2^{-1 / 3} A^{-6}, A$ being the Glaisher constant $(A=1.2824 \ldots)$. It should be mentioned that we obtained the value of the constant in (5.7) comparing the behaviour of correlator (1.1) at $T \rightarrow 0, x \rightarrow+\infty$ with the behaviour at $T=0, x \rightarrow+\infty$ using the correspondence between these asymptotics known in the conformal field theory [7].

\section{Conclusion}

We demonstrated that by applying the inverse scattering method to the integrable nonlinear system describing temperature correlators of the impenetrable bosons permits us to obtain the most interesting information from the physical point of view, namely, to calculate the long-distance asymptotics of correlators. It should be emphasized that it is done directly in the model considered (the conformal hypothesis was used only to determine constant $\rho_{\infty}$ in (5.7)). Corresponding results for zero-temperature correlators were obtained in paper [8]; at $T=0$, the correlators depend on the product $z h^{1 / 2}$ only and are described by ordinary differential equations.

In paper [1] the multi-point temperature correlators were also considered. The matrix Riemann problem corresponding to this case is given in the Appendix.

\section{Appendix}

In Sect. 7 of paper [1] the multi-point correlators $\left\langle\psi_{1}^{+}\left(z_{1}^{+}\right) \cdots \psi_{N}^{+}\left(z_{N}\right) \psi_{1}\left(z_{1}^{-}\right) \cdots\right.$ $\left.\psi_{N}\left(z_{N}^{-}\right)\right\rangle_{T}$ were shown to be described by a multidimensional integrable system and corresponding partial differential equations in $(2 N+1)$ variables $t, x_{1}, \ldots x_{2 N}$ $\left(t=h / T, x=z T^{1 / 2}\right)$ were given. Below the matrix Riemann problem for this system is briefly discussed. It is supposed that the reader knows the contents of Sect. 7 of [1]. References to the corresponding formulae will be given as (I.7.1) (which means Eq. (7.1) of [1]).

The MRP for multi-point correlators is a natural generalization of the MRP considered above in Sect. 2. Now $\chi(\lambda)$ is a $(2 N \times 2 N)$-matrix and the conjugating 
matrix $G(\lambda)$ in (2.2) is defined as

$$
\begin{aligned}
G_{m n}(\lambda) & =\delta_{m n}+(-1)^{n} \pi \gamma e_{m}^{+}(\lambda) e_{n}^{-}(\lambda) \quad(m, n=1, \ldots, 2 N), \\
e_{m}^{ \pm}(\lambda) & =\sqrt{\vartheta(\lambda)} \exp \left\{ \pm i x_{m} \lambda\right\} .
\end{aligned}
$$

Matrix $\chi(\lambda)$ at $\lambda \rightarrow \infty$ can be expanded as

$$
\chi(\lambda)=I+\left(\Psi_{1} / \lambda\right)+\cdots,
$$

where functions $\Psi_{i}$ do not depend on $\lambda$. Representing

$$
\left(\Psi_{1}\right)_{m n}=(1 / 2 i) V_{l m},
$$

one has due to the symmetry properties of $G(\lambda)$ that $V_{l m}=V_{l m}^{*}=(-1)^{l+m} V_{l m}$. Considering the matrix

$$
\Psi(\lambda, x, t)=\chi(\lambda, x, t) \exp \{i \lambda J\}, \quad J_{m n}=\delta_{m n} x_{n},
$$

one calculates the corresponding conjugating matrix

$$
G_{0}(\lambda)=\delta_{m n}+(-1)^{n} \pi \gamma \vartheta(\lambda)
$$

which satisfies equations $\partial_{n} G_{0}(\lambda)=0\left(\partial_{n} \equiv \partial / \partial x_{n}\right)$ and $\left(2 \lambda \partial_{t}+\partial_{\lambda}\right) G_{0}(\lambda)=0$. Hence one obtains as in Sect. 2 that

$$
\left\{\begin{array}{c}
\left(\partial_{n} \Psi\right) \Psi^{-1}=i \lambda J_{n}+U_{n} \\
{\left[\left(2 \lambda \partial_{t}+\partial_{\lambda}\right) \Psi\right] \Psi^{-1}=V_{0}}
\end{array}\right.
$$

with matrices $U_{n}$ and $V_{0}$ equal to

$$
\left\{\begin{array}{c}
\left(U_{n}\right)_{l m}=-\frac{1}{2} \delta_{n l} V_{m l}+\frac{1}{2} V_{n l} V_{n l} \delta_{n m} \\
\left(V_{0}\right)_{l m}=i x_{l} \delta_{l m}-i \partial_{t} V_{m l}
\end{array}\right.
$$

Thus function $\Psi(\lambda)$ is a matrix solution of the zero curvature representation (A.6) which is similar to the corresponding representation (I.7.12), (I.7.13) for the vector-function $f_{m}^{+}$.

The compatibility conditions lead just to nonlinear differential equation (I.7.14)-(I.7.18) for "potentials" $V_{l m}(x, t)$.

One can now prove that potentials $V_{l m}$ introduced here (A.3) not only satisfy the same equations as potentials $V_{l m}$ in paper [1] (see (I.7.7)) but are the same. As in Sect. 2 the proof is based on rewriting the system of singular integral equations (2.11) for functions $\hat{\chi}(\lambda)$ :

$$
\begin{array}{rlrl}
\hat{\chi}(\lambda) & =\chi^{+}(\lambda) E(\lambda), \\
E_{n m} & =1, & & =1, \ldots, 2 N-1, \\
E_{n m} & =0, & & =1, \ldots, 2 N ; m \neq n, m=1, \ldots, 2 N-1, \\
E_{n, 2 N} & =e_{n}^{+}(\lambda), & & n=1, \ldots, 2 N .
\end{array}
$$

For functions $\hat{\chi}_{n, 2 N}(\lambda)$ one obtains integral equations

$$
\hat{\chi}_{n, 2 N}(\lambda)=e^{+}(\lambda)+\gamma \int_{-\infty}^{\infty} K(\lambda, \mu) \hat{\chi}_{n, 2 N}(\mu) d \mu,
$$


with kernel $K$ equal to

$$
K(\lambda, \mu)=\sum_{m=1}^{2 N}(-1)^{m}[2 i(\lambda-\mu)]^{-1} e_{m}^{+}(\lambda) e_{m}^{-}(\lambda)
$$

which are just Eqs. (I.7.6) for functions $f^{+}(\lambda)$. Generalizing now the corresponding arguments given in Sect. 2 in the case $N=1$, one comes to the representation for $V_{l m}$,

$$
V_{l m}=(-1)^{l} \gamma \int_{-\infty}^{\infty} e_{l}^{-}(\mu) f_{m}^{+}(\mu) d \mu
$$

which is just the definition of potentials in [1].

So the matrix Riemann problem constructed is indeed the MRP of the inverse scattering method for an integrable system describing multi-point correlators.

Acknowledgements. The authors would like to thank L. D. Faddeev and A. Verjovsky for discussions. One of the authors (A.G.I.) would like to thank Professor Abdus Salam, the International Atomic Energy Agency and UNESCO for hospitality at the International Centre for Theoretical Physics, Trieste.

\section{References}

1. Its, A. R., Izergin, A. G., Korepin, V. E.: Temperature correlators of the impenetrable Bose gas as an integrable system. Commun. Math. Phys. 129, 205-222 (1990)

2. Faddeev, L. D., Takhtajan, L. A.: Hamiltonian methods in the theory of solitons. Berlin, Heidelberg, New York: Springer 1987

3. Manakov, S. V.: Nonlinear Fraunhofer diffraction. Zh. Exp. Teor. Fiz. 65, 1392-1403 (1973)

4. Gokhberg, I. Z., Krein, M. P.: Systems of integral equations on a half-axis with kernels depending on difference of variables. Usp. Matem. Nauk 13, 3-72 (1958)

5. Its, A. R., Izergin, A. G., Korepin, V. E.: Correlation radius for one dimensional impenetrable bosons. ICTP, Trieste, preprint IC/89/107 (1989) (submitted to Phys. Lett. A).

6. Vaidiya, H. G., Tracy, C. A.: One-particle reduced density matrix of impenetrable bosons in one dimension at zero temperature. J. Math. Phys. 20, 2291-2303 (1979)

7. Affleck, I.: Universal term in the free energy at a critical point and the conformal anomaly. Phys. Rev. Lett. 56, 746-748 (1986)

8. Jimbo, A., Miwa, T., Môri, Y., Sato, M.: Density matrix of an impenetrable Bose gas and the fifth Painlevé transcendent. Physica 1D, 80-158 (1980)

Communicated by A. Jaffe

Received October 5, 1989 